\title{
Targeted metabolic profiling and PRM analysis of proteins revealed impaired polyunsaturated fatty acid metabolism and GTP
}

metabolism in brainstem of spontaneously hypertensive rats

Wenbin Zhou ${ }^{\mathrm{a}, \mathrm{c}, 1}$, Bangjie Zhu ${ }^{\mathrm{a}, \mathrm{b}, 1}$, Fang Kou ${ }^{\mathrm{a}, 1}$, Shenglan $\mathrm{Qi}^{\mathrm{a}}$, Chunming $\mathrm{Lv}^{\mathrm{c}}$, Yu Cheng ${ }^{\mathrm{a}, \mathrm{b}^{*}}$, Hai Wei ${ }^{\mathrm{a}^{*}}$

${ }^{a}$ Institute of Interdisciplinary Integrative Medicine Research, Shanghai University of Traditional Chinese Medicine, Shanghai 201203, China

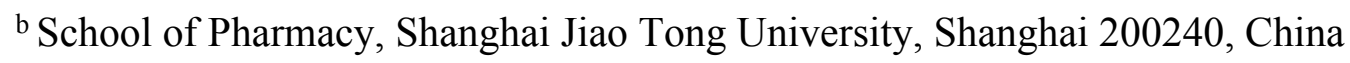

${ }^{\mathrm{c}}$ Shanghai Zhulian Intelligent Technology Ltd. Co., Shanghai 201323, China

${ }^{1}$ These authors contributed equally to this paper.

${ }^{*}$ Correspondence to: Professor Hai Wei, Email: wei_hai@hotmail.com, or Dr. Yu Cheng, E-mail: ycheng_16@163.com, Institute of Interdisciplinary Integrative Medicine Research, Shanghai University of Traditional Chinese Medicine, Shanghai 201203, China. 
Supplementary information 1. Standard protocol for the experiments.

Supplementary Table S1. Quantitative parameters of 32 metabolites of nucleotides and energy metabolism.

Supplementary Table S2. Quantitative parameters of 40 fatty acids

Supplementary Table S3. Fatty acids with VIP (variable importance in projection) $>1$ in OPLS-DA analysis based on 40 fatty acids between SHRs and normotensive rats.

Supplementary Table S4. The precision values of the five proteins in PRM methods.

Supplementary Figure S1. (a, b). RSD (\%) of the quality control (QC) samples (fatty acids, nucleotides and energy metabolism).

Supplementary Figure S2. Plots of PCA and OPLS-DA analysis between SHRs and normotensive rats. a. PCA based on 40 fatty acids; b. OPLS-DA based on 40 fatty acids; c. PCA based on 32 chemicals of nucleotides and energy metabolism; d. OPLS-DA based on 32 chemicals of nucleotides and energy metabolism.

Supplementary Figure S3. The chromatograms of PRM analysis for the five proteins. 
Supplementary information 1. Standard protocol for the experiments.

The experiments of targeted metabolic profiling of fatty acids, nucleotides and energy metabolism and parallel reaction monitoring (PRM) analysis of five proteins depend on good quality control in the whole performance line of sample collection, sample storage, sample preparation, sample analysis using Gas/Liquid Chromatograph coupled with mass spectrometer or tandem mass spectrometer (GC/MS, LC-MS/MS). We prepared this standard protocol for our experiments to guarantee the data quality, according to the experience in our previous studies ${ }^{1-3}$.

\section{Targeted metabolic profiling of fatty acids}

The targeted metabolic profiling of 40 fatty acids were modified according to previous studies ${ }^{2,4}$.

\section{Tissue preparation}

The brainstem tissue $50 \mathrm{mg}$ was thawed in ice, and $1 \mathrm{~mL}$ chloroform methanol solution were added. After ultrasonic for $30 \mathrm{~min}$, supernatant was transferred, and $2 \mathrm{ml}$ sulfuric acid - methanol solution (1\%) was added. Then the mixture was taken on the water bath with $80{ }^{\circ} \mathrm{C}$ for half-hour methyl esterification. Then $1 \mathrm{ml}$ of $\mathrm{n}$-hexane was added for extraction and $5 \mathrm{ml}$ water was added for washing. The supernatant $500 \mu \mathrm{L}$ was transferred and the internal standard of $25 \mu \mathrm{L}$ salicylic acid methyl was spiked into it. The mixture was transferred to the sample bottle and analyzed by GC-MS.

\section{Calibration solution}

Standard solutions of 40 chemicals of fatty acids were freshly prepared in different concentration levels (10 points) of $0.5 \mathrm{mg} / \mathrm{L}, 1 \mathrm{mg} / \mathrm{L}, 5 \mathrm{mg} / \mathrm{L}, 10 \mathrm{mg} / \mathrm{L}, 25 \mathrm{mg} / \mathrm{L}, 50$ 
$\mathrm{mg} / \mathrm{L}, 100 \mathrm{mg} / \mathrm{L}, 250 \mathrm{mg} / \mathrm{L}, 500 \mathrm{mg} / \mathrm{L}, 1000 \mathrm{mg} / \mathrm{L}$. Salicylic acid was used as the internal standard (500 ppm, $25 \mu \mathrm{L}$ internal standard was spiked into $500 \mu \mathrm{L}$ standard mixture).

\section{GC-MS analysis}

The targeted metabolic profiling analysis of 40 fatty acids was performed with an Agilent 7890A/5975C (Agilent Technologies) gas chromatography coupled with a mass spectrometer, using an Agilent DB-WAX capillary column $(30 \mathrm{~m} \times 0.25 \mathrm{~mm}$ ID $\times 0.25 \mu \mathrm{m})$. The temperature programming was as follows: the initial temperature was $50^{\circ} \mathrm{C}$ and remained as such for $3 \mathrm{~min}$. The temperature increased at $10^{\circ} \mathrm{C} / \mathrm{min}$ up to $220^{\circ} \mathrm{C}$ and remained there for $5 \mathrm{~min}$. The carrier gas was helium, and the carrier gas velocity was $1.0 \mathrm{~mL} / \mathrm{min}$. The temperatures of the injection port and transmission line were $280{ }^{\circ} \mathrm{C}$ and $250{ }^{\circ} \mathrm{C}$, respectively. The electron bombardment ionization (EI) source, SIM scanning mode, and electron energy were $70 \mathrm{eV}$. Injection volume was 1 $\mu \mathrm{L}$ in split mode with a split rate of 10:1. Injections of QC were inserted during the analysis sequence. Mixture of chemical standard solutions was used to align the retention time of the peaks.

\section{Data processing}

Data acquisition and processing were accomplished using MSD ChemStation software.

\section{Targeted metabolic profiling of nucleotides and energy metabolism}

The targeted metabolic profiling of 32 chemicals of nucleotides and energy 
metabolism were modified according to previous studies ${ }^{1}$.

\section{Tissue preparation}

The brainstem tissue $60 \mathrm{mg}$ was homogenized with $200 \mu \mathrm{L}$ water, and $800 \mu \mathrm{L}$ cold solvent mixture of methanol and acetonitrile $(1: 1, \mathrm{v} / \mathrm{v})$ were added to the mixture. Then the mixture was taken into sonication on ice for $30 \mathrm{~min}$, and repeated twice. After incubation at $-20^{\circ} \mathrm{Cfor}$ one hour and centrifugation at $13000 \mathrm{~g}$ at $4^{\circ} \mathrm{C}$ for $20 \mathrm{~min}$, the supernatant was transferred for LC-MS/MS analysis.

\section{Calibration solution}

Standard solutions of 32 chemicals of nucleotides and energy metabolism were freshly prepared in different concentration levels (11 points) of $0.1 \mu \mathrm{mol} / \mathrm{L}, 0.5 \mu \mathrm{mol} / \mathrm{L}$, $1 \mu \mathrm{mol} / \mathrm{L}, 5 \mu \mathrm{mol} / \mathrm{L}, 10 \mu \mathrm{mol} / \mathrm{L}, 25 \mu \mathrm{mol} / \mathrm{L}, 50 \mu \mathrm{mol} / \mathrm{L}, 100 \mu \mathrm{mol} / \mathrm{L}, 250 \mu \mathrm{mol} / \mathrm{L}, 500$ $\mu \mathrm{mol} / \mathrm{L}, 1000 \mu \mathrm{mol} / \mathrm{L}$. The isotope compound succinic acid (D6, 98\%, DLM-831-5, CIL) was used as internal standard $(10 \mathrm{mmol}, 10 \mu \mathrm{L}$ internal standard was spiked into $100 \mu \mathrm{L}$ standard mixture).

\section{LC-MS/MS analysis}

The targeted metabolic profiling analysis of nucleotides and energy metabolism was performed with an UHPLC (1290 Infinity LC, Agilent Technologies) coupled with a tandem mass spectrometer (AB Sciex 5500 QTRAP), using a $2.1 \mathrm{~mm} \times 100 \mathrm{~mm}$ ACQUIY UPLC BEH Amide $1.7 \mu \mathrm{m}$ column (Waters, Ireland) in ESI negative mode. Column temperature was set to $45^{\circ} \mathrm{C}$. The mobile phase A was $10 \mathrm{mM}$ ammonium acetate in water, and B 100\% acetonitrile. The gradient was initiated with $90 \% \mathrm{~B}$ linearly reduced to $40 \%$ in $18 \mathrm{~min}$, and then increased to $90 \%$ in $0.1 \mathrm{~min}$ and kept a 
final re-equilibration period of $5 \mathrm{~min}$. Flow rate was $300 \mu \mathrm{L} / \mathrm{min}$, and the injection volume was $2 \mu \mathrm{L}$. Injections of quality control (QC) were inserted during the analysis sequence. Mixture of chemical standard solutions was used to align the retention time of the peaks.

The ESI source were set with conditions as follows: Ion Source Gas1 (Gas1), 45 psi; Ion Source Gas2 (Gas2), 45 psi; curtain gas (CUR), 30 psi; source temperature, $350^{\circ} \mathrm{C}$; Ion Spray Voltage Floating (ISVF), $4500 \mathrm{~V}$. The mass spectrometer operated with a dwell time of $200 \mathrm{~ms} / \mathrm{spectrum}$.

\section{Data processing}

Data acquisition and processing were accomplished using Multiquant software.

\section{Parallel reaction monitoring (PRM) analysis}

Five proteins, which were relative to the altered metabolic pathways in the analysis of proteomic profiling, were analyzed by PRM analysis including 2,4-dienoyl-CoA reductase, mitochondrial (DECR), protein-arginine deiminase type-2 (PADI2), transforming protein RhoA (RHOA), guanine nucleotide-binding protein G(I)/G(S)/G(T) subunit beta-1 (GNB1), and Rho-related GTP-binding protein RhoB (RHOB).

\section{PRM method development for the selected proteins}

The PRM analysis methods for the five proteins were developed and precision of three injections was validated.

Protein extraction and trypsin digestion 
Protein extraction and trypsin digestion were modified according to our previous study and previous literature ${ }^{3,5}$.

\section{Nano-HPLC-MS/MS analysis}

Nano-HPLC-MS/MS analysis were described in previous study ${ }^{3}$. Peptides of $2 \mu \mathrm{g}$ were spiked with 20 fmol standard (PRTC: GISNEGQNASIK). The sample was separated on an Easy nLC 1200 System (Thermo Fisher Scientific, United States) using a column of $300 \mu \mathrm{m}^{*} 150 \mathrm{~mm}, 1.7 \mu \mathrm{m}-\mathrm{C} 18$. Buffer A was of $0.1 \%(\mathrm{v} / \mathrm{v})$ formic acid and B of $0.1 \%(\mathrm{v} / \mathrm{v})$ formic acid in $84 \%(\mathrm{v} / \mathrm{v})$ acetonitrile. Flow rate was set to $300 \mathrm{~nL} / \mathrm{min}$. The gradient of B was set as follows: 5\% B to $10 \% \mathrm{~B}$ in $2 \mathrm{~min}, 10-30 \%$ in $43 \mathrm{~min}, 30$ $-100 \%$ in $10 \mathrm{~min}$ and then $100 \%$ for another $5 \mathrm{~min}$. The eluted peptides were analyzed on a Q-Exactive HF mass spectrometer (Thermo Fisher Scientific, United States) in positive ion mode. The ions were first subjected to one survey scan in the $\mathrm{m} / \mathrm{z}$ range of 300 to 1,800 at a mass resolution of 60,000 at $\mathrm{m} / \mathrm{z} 200$. Automatic gain control target and maximum inject time were set to $3 \times 106$ and $200 \mathrm{~ms}$, respectively. The full MS1 scan was followed by 20 MS2 scans at a resolution of 17500 at m/z 200. Precursor ions were fragmented at normalized collision energy of $27 \mathrm{eV}$. The obtained raw PRM data were imported into Skyline (version 3.5.0) for analysis.

\section{Reference}

1. Zhu, B.; Wei, H.; Wang, Q.; Li, F.; Dai, J.; Yan, C.; Cheng, Y., A simultaneously quantitative method to profiling twenty endogenous nucleosides and nucleotides in cancer cells using UHPLCMS/MS. Talanta 2018, 179, 615-623.

2. Cheng, Y.; Xie, G.; Chen, T.; Qiu, Y.; Zou, X.; Zheng, M.; Tan, B.; Feng, B.; Dong, T.; He, P.; Zhao, L.; Zhao, A.; Xu, L. X.; Zhang, Y.; Jia, W., Distinct urinary metabolic profile of human colorectal cancer. Journal of proteome research 2012, 11, (2), 1354-63.

3. Cheng, Y.; Sun, D.; Zhu, B.; Zhou, W.; Lv, C.; Kou, F.; Wei, H., Integrative Metabolic and 
Proteomic Profiling of the Brainstem in Spontaneously Hypertensive Rats. Journal of proteome research 2020, 19, (10), 4114-4124.

4. Liu, Y.; Zeng, L.; Yang, Y.; Chen, C.; Wang, D.; Wang, H., Acyl-CoA thioesterase 1 prevents cardiomyocytes from Doxorubicin-induced ferroptosis via shaping the lipid composition. Cell death \& disease 2020, 11, (9), 756.

5. Sun, D.; Cheng, Y.; Zhou, D.; Liu, T.; Chen, S.; Liang, J.; Tang, C.; Lai, X., Quantitative proteome of medulla oblongata in spontaneously hypertensive rats. Journal of proteome research 2013, 12, (1), $390-5$. 
Supplementary Table S1. Quantitative parameters of 32 metabolites of nucleotides and energy metabolism.

\begin{tabular}{|c|c|c|c|c|c|}
\hline Component Name & Mass Info & $\begin{array}{c}\text { Retention } \\
\text { Time }\end{array}$ & Linear & $\mathbf{R}^{\wedge} \mathbf{2}$ & $\begin{array}{c}\text { Linear } \\
\text { range } \\
(\mu \mathrm{mol} / \mathrm{L})\end{array}$ \\
\hline 3-Phospho-D-glycerate & $185.0 / 97.0$ & 7.94 & $y=0.66274 x+-1.03427$ & 0.9994 & $10-500$ \\
\hline Acetyl coenzyme A (Acetyl-CoA) & $808.2 / 408.1$ & 6.03 & $y=0.58676 x+0.01215$ & 0.9956 & $0.1-500$ \\
\hline Adenosine 5'-triphosphate (ATP) & $506.0 / 159.0$ & 8.70 & $y=2.30692 x+-5.48047$ & 0.9986 & $2.5-500$ \\
\hline $\mathrm{ADP}$ & $426.0 / 79.0$ & 7.55 & $y=3.04980 x+-0.56916$ & 0.9980 & $0.1-500$ \\
\hline alpha-ketoglutarate & $145.2 / 101.1$ & 3.94 & $\mathrm{y}=0.30032 \mathrm{x}+3.10058$ & 0.9994 & $10-500$ \\
\hline Adenosine monophosphate & $346.1 / 79.0$ & 6.24 & $y=2.75700 x+0.77972$ & 1.0000 & $0.5-100$ \\
\hline Beta-D-Fructose 6-phosphate & $259.1 / 97.0$ & 7.39 & $\mathrm{y}=0.82959 \mathrm{x}+0.63522$ & 0.9843 & $0.1-100$ \\
\hline cis-Aconitate & $173.1 / 85.1$ & 7.02 & $y=11.48134 x+-2.57467$ & 0.9942 & $0.1-250$ \\
\hline Citrate & $191.1 / 87.1$ & 7.86 & $y=0.76717 x+20.01039$ & 0.9954 & $10-500$ \\
\hline Cyclic AMP & $328.0 / 134.0$ & 1.99 & $y=10.74684 x+0.22339$ & 0.9964 & $0.1-500$ \\
\hline D-Fructose 1,6-bisphosphate & $339.1 / 97.0$ & 11.04 & $y=1.58145 x+-80.90216$ & 0.9992 & $25-500$ \\
\hline D-Glucose 6-phosphate & $259.0 / 97.0$ & 8.33 & $y=1.27889 x+-3.18258$ & 0.9968 & $0.1-100$ \\
\hline Dihydroxyacetone phosphate (glycerone-p) & $169.1 / 97.0$ & 6.28 & $y=0.31395 x+-4.75186$ & 0.9956 & $0.1-500$ \\
\hline Flavin mononucleotide (FMN) & $455.1 / 97.0$ & 5.15 & $y=1.13399 x+-0.06821$ & 0.9958 & $0.1-100$ \\
\hline Fumarate & $115.1 / 71.1$ & 4.25 & $y=0.09885 x+0.31663$ & 0.9984 & $0.1-500$ \\
\hline GMP & $362.1 / 79.1$ & 7.84 & $y=1.62605 x+2.12382$ & 0.9942 & $0.5-100$ \\
\hline Guanosine 5'-diphosphate (GDP) & $442.0 / 79.0$ & 9.17 & $y=2.72130 x+-13.38106$ & 0.9974 & $2.5-250$ \\
\hline Guanosine 5'-triphosphate (GTP) & $522.0 / 159.1$ & 10.35 & $y=0.61564 x+-6.63200$ & 0.9996 & $5-250$ \\
\hline Isocitrate & $191.0 / 73.0$ & 7.78 & $y=1.01410 x+-0.03849$ & 0.9976 & $0.1-100$ \\
\hline Lactate & $89.2 / 43.0$ & 1.82 & $y=0.00672 x+0.17952$ & 0.9996 & $10-500$ \\
\hline L-Malic acid & $133.0 / 115.1$ & 5.17 & $y=7.59422 x+-0.74819$ & 0.9994 & $1-100$ \\
\hline
\end{tabular}




\begin{tabular}{|c|c|c|c|c|c|}
\hline NAD & $662.1 / 540.0$ & 6.44 & $y=0.92356 x+0.50661$ & 0.9972 & $0.1-100$ \\
\hline NADP & $742.1 / 620.0$ & 10.15 & $y=3.51262 x+-2.82875$ & 0.9978 & $0.1-100$ \\
\hline NADPH & $744.1 / 408.0$ & 10.15 & $y=0.02192 x+-0.00607$ & 0.9998 & $0.25-100$ \\
\hline Oxaloacetate & $131.1 / 87.1$ & 4.56 & $y=0.03930 x+0.11447$ & 0.9994 & $0.1-500$ \\
\hline Phosphoenolpyruvate & $167.1 / 79.0$ & 7.64 & $y=1.09820 x+0.38349$ & 0.9958 & $0.25-500$ \\
\hline Pyruvate & $87.1 / 43.1$ & 1.37 & $y=0.00153 x+-0.04615$ & 0.9956 & $10-500$ \\
\hline \multicolumn{6}{|c|}{ Reduced nicotinamide adenine dinucleotide } \\
\hline$(\mathrm{NADH})$ & $664.1 / 408.0$ & 6.46 & $y=0.00495 x+-0.00144$ & 0.9990 & $0.1-100$ \\
\hline Succinate & $117.1 / 73.0$ & 4.73 & $y=1.99143 x+0.98726$ & 0.9998 & $0.1-500$ \\
\hline Succinyl coenzyme A (Succinyl-CoA) & $866.1 / 408.1$ & 9.13 & $y=0.00553 x+-0.20218$ & 0.9950 & $10-500$ \\
\hline Thiamine pyrophosphate (TPP) & $423.0 / 302.0$ & 9.10 & $y=1.55574 x+-0.75409$ & 0.9992 & $0.1-100$ \\
\hline Glutaryl-CoA & $880.1 / 407.9$ & 8.88 & $y=1.05784 x+-4.55258$ & 0.9984 & $0.1-500$ \\
\hline
\end{tabular}


Supplementary Table S2. Quantitative parameters of 40 fatty acids

\begin{tabular}{lllcc}
\hline Name & Abbreviation & Linear equation & \multicolumn{2}{c}{$\begin{array}{c}\text { Linear range } \\
(\boldsymbol{\mu g} / \mathbf{m l})\end{array}$} \\
\hline butyrate & $\mathrm{C} 4: 0$ & $\mathrm{y}=0.676115 * \mathrm{x}+0.010459$ & 0.9996 & $0.002-50$ \\
hexanoate & $\mathrm{C} 6: 0$ & $\mathrm{y}=2.145062 * \mathrm{x}+2.400182 \mathrm{E}-004$ & 0.9997 & $0.002-50$ \\
octanoate & $\mathrm{C} 8: 0$ & $\mathrm{y}=2.808091 * \mathrm{x}+3.552830 \mathrm{E}-004$ & 0.9997 & $0.002-50$ \\
decanoate & $\mathrm{C} 10: 0$ & $\mathrm{y}=2.974875 * \mathrm{x}+4.407884 \mathrm{E}-004$ & 0.9997 & $0.004-100$ \\
undecanoate & $\mathrm{C} 11: 0$ & $\mathrm{y}=2.938498 * \mathrm{x}+3.125274 \mathrm{E}-004$ & 0.9998 & $0.002-50$ \\
dodecanoate & $\mathrm{C} 12: 0$ & $\mathrm{y}=2.924615 * \mathrm{x}+3.922994 \mathrm{E}-004$ & 0.9998 & $0.004-100$ \\
tridecanoate & $\mathrm{C} 13: 0$ & $\mathrm{y}=2.774600 * \mathrm{x}+3.058208 \mathrm{E}-004$ & 0.9997 & $0.002-50$ \\
myristate & $\mathrm{C} 14: 0$ & $\mathrm{y}=2.578346 * \mathrm{x}+0.015441$ & 0.9996 & $0.002-50$ \\
myristoleate & $\mathrm{C} 14: 1 \mathrm{~N} 5$ & $\mathrm{y}=1.093605 * \mathrm{x}-6.418088 \mathrm{E}-004$ & 0.9993 & $0.002-50$ \\
pentadecanoate & $\mathrm{C} 15: 0$ & $\mathrm{y}=2.534515 * \mathrm{x}+1.383307 \mathrm{E}-004$ & 0.9997 & $0.002-50$ \\
cis-10-pentadecenoate & $\mathrm{C} 15: 1 \mathrm{~N} 5$ & $\mathrm{y}=0.979738 * \mathrm{x}+1.153024 \mathrm{E}-004$ & 0.9998 & $0.002-50$ \\
palmitate & $\mathrm{C} 16: 0$ & $\mathrm{y}=2.542880 * \mathrm{x}+0.071060$ & 0.9997 & $0.004-100$ \\
palmitoleate & $\mathrm{C} 16: 1 \mathrm{~N} 7$ & $\mathrm{y}=0.765158 * \mathrm{x}+0.005247$ & 0.9997 & $0.002-50$ \\
heptadecanoate & $\mathrm{C} 17: 0$ & $\mathrm{y}=2.399196 * \mathrm{x}-8.808896 \mathrm{E}-004$ & 0.9997 & $0.004-100$ \\
cis-10-heptadecenoate & $\mathrm{C} 17: 1 \mathrm{~N} 7$ & $\mathrm{y}=0.746499 * \mathrm{x}+1.371566 \mathrm{E}-004$ & 0.9997 & $0.002-50$ \\
stearate & $\mathrm{C} 18: 0$ & $\mathrm{y}=2.435727 * \mathrm{x}+0.084298$ & 0.9996 & $0.004-100$ \\
elaidate & $\mathrm{C} 18: 1 \mathrm{TN} 9$ & $\mathrm{y}=0.775054 * \mathrm{x}-0.004505$ & 0.9996 & $0.002-50$ \\
oleate & $\mathrm{C} 18: 1 \mathrm{~N} 9$ & $\mathrm{y}=0.716432 * \mathrm{x}+0.100592$ & 0.9995 & $0.004-100$ \\
linolelaidate & $\mathrm{C} 18: 2 \mathrm{TTN} 6$ & $\mathrm{y}=0.964511 * \mathrm{x}-0.020342$ & 0.9996 & $0.002-50$ \\
linoleate & $\mathrm{C} 18: 2 \mathrm{~N} 6$ & $\mathrm{y}=0.956557 * \mathrm{x}-0.026392$ & 0.9996 & $0.002-50$ \\
$\gamma$-linolenate & $\mathrm{C} 18: 3 \mathrm{~N} 6$ & $\mathrm{y}=0.781160 * \mathrm{x}+3.118248 \mathrm{E}-004$ & 0.9999 & $0.004-100$ \\
& & &
\end{tabular}




\begin{tabular}{|c|c|c|c|c|c|}
\hline linolenate & $\mathrm{C} 18: 3 \mathrm{~N} 3$ & $\mathrm{y}=1.046795 * \mathrm{x}$ & -0.004403 & 0.9998 & $0.002-50$ \\
\hline arachidate & $\mathrm{C} 20: 0$ & $\mathrm{y}=1.870038 * \mathrm{x}$ & $+1.526439 \mathrm{E}-004$ & 0.9998 & $0.004-100$ \\
\hline cis-11-Eicosenoic acid & C20:1N9 & $\mathrm{y}=0.827732 * \mathrm{x}$ & -0.007419 & 1.0000 & $0.002-50$ \\
\hline cis-11,14-Eicosadienoic acid & $\mathrm{C} 20: 2 \mathrm{~N} 6$ & $y=0.914886 * x$ & -0.023660 & 0.9995 & $0.002-50$ \\
\hline heneicosanoate & $\mathrm{C} 21: 0$ & $\mathrm{y}=1.552001 * \mathrm{x}$ & +0.001375 & 0.9999 & $0.002-50$ \\
\hline cis-8,11,14-Eicosatrienoic acid & $\mathrm{C} 20: 3 \mathrm{~N} 6$ & $\mathrm{y}=0.878719 * x$ & -0.029676 & 0.9995 & $0.002-50$ \\
\hline arachidonate & C20:4N6 & $\mathrm{y}=0.948662 * \mathrm{x}$ & -0.047859 & 0.9995 & $0.002-50$ \\
\hline \multicolumn{6}{|l|}{ cis-11,14,17-Eicosatrienoic } \\
\hline acid & $\mathrm{C} 20: 3 \mathrm{~N} 3$ & $\mathrm{y}=1.123874 * \mathrm{x}$ & -0.064862 & 0.9995 & $0.002-50$ \\
\hline behenate & $\mathrm{C} 22: 0$ & $\mathrm{y}=1.602850 * \mathrm{x}$ & $-2.476369 \mathrm{E}-004$ & 0.9995 & $0.004-100$ \\
\hline \multicolumn{6}{|l|}{ cis-5,8,11,14,17- } \\
\hline Eicosapentaenoic acid & $\mathrm{C} 20: 5 \mathrm{~N} 3$ & $\mathrm{y}=1.080144 * \mathrm{x}$ & -0.010530 & 1.0000 & $0.002-50$ \\
\hline erucate & C22:1N9 & $\mathrm{y}=0.940492 * \mathrm{x}$ & -0.008849 & 0.9999 & $0.002-50$ \\
\hline cis-13,16-Docosadienoic acid & $\mathrm{C} 22: 2 \mathrm{~N} 6$ & $\mathrm{y}=0.548524 * \mathrm{x}$ & $+3.968627 \mathrm{E}-005$ & 0.9994 & $0.002-50$ \\
\hline tricosanoate & $\mathrm{C} 23: 0$ & $\mathrm{y}=1.880202 * \mathrm{x}$ & -0.008220 & 0.9999 & $0.002-50$ \\
\hline docosatetraenoate & $\mathrm{C} 22: 4 \mathrm{~N} 6$ & $\mathrm{y}=1.009597 * \mathrm{x}$ & -0.025842 & 0.9992 & $0.002-50$ \\
\hline docosapentaenoate & $\mathrm{C} 22: 5 \mathrm{~N} 6$ & $\mathrm{y}=1.021545 * \mathrm{x}$ & -0.025451 & 0.9992 & $0.002-50$ \\
\hline tetracosanoate & $\mathrm{C} 24: 0$ & $\mathrm{y}=1.786569 * \mathrm{x}$ & -0.018456 & 0.9998 & $0.004-100$ \\
\hline docosapentaenoate & $\mathrm{C} 22: 5 \mathrm{~N} 3$ & $\mathrm{y}=1.026703 * \mathrm{x}$ & -0.038717 & 0.9999 & $0.002-50$ \\
\hline cis-15-tetracosenoate & C24:1N9 & $\mathrm{y}=1.041510 * \mathrm{x}$ & -0.048647 & 0.9997 & $0.002-50$ \\
\hline \multicolumn{6}{|l|}{ cis-4,7,10,13,16,19- } \\
\hline Docosahexaenoic acid & $\mathrm{C} 22: 6 \mathrm{~N} 3$ & $\mathrm{y}=1.096107 * \mathrm{x}$ & -0.067056 & 0.9995 & $0.002-50$ \\
\hline
\end{tabular}


Supplementary Table S3. Fatty acids with VIP (variable importance in projection) $>1$ in OPLS-DA analysis based on 40 fatty acids between SHRs and normotensive rats.

\begin{tabular}{lll}
\hline Fatty Acid & Abbreviation & VIP total \\
\hline palmitoleate & C16:1N7 & 1.154775 \\
docosatetraenoate & C22:4N6 & 1.499873 \\
stearate & $\mathrm{C} 18: 0$ & 2.665403 \\
palmitate & $\mathrm{C} 16: 0$ & 2.490727 \\
oleate & $\mathrm{C} 18: 1 \mathrm{~N} 9$ & 2.323516 \\
cis-11-Eicosenoic acid & $\mathrm{C} 20: 1 \mathrm{~N} 9$ & 1.642971 \\
arachidonate & $\mathrm{C} 20: 4 \mathrm{~N} 6$ & 2.178665 \\
cis-15-tetracosenoate & $\mathrm{C} 24: 1 \mathrm{~N} 9$ & 1.919697 \\
\hline
\end{tabular}


Supplementary Table S4. The precision values of the five proteins in PRM methods.

\begin{tabular}{lccccccc}
\hline Name & IPI access & Unique peptide & Run-1 & Run-2 & Run-3 & AVERAGE & RSD \\
\hline DECR1 & IPI: IPI00213659 & FNIIQPGPIK & 2638700 & 2468400 & 2522600 & 2543233 & $3.42 \%$ \\
PADI2 & IPI: IPI00202307 & ILSNESLTQENQYFQR & 1040500 & 988540 & 1101000 & 1043347 & $5.39 \%$ \\
RHOA & IPI: IPI00201699 & LVIVGDGACGK & 7771000 & 8826300 & 7068000 & 7888433 & $11.22 \%$ \\
RHOB & IPI: IPI00210815 & EVFETATR & 73494000 & 87028000 & 70594000 & 77038667 & $11.39 \%$ \\
GNB1 & IPI: IPI00212655 & LFVSGACDASAK & 26935000 & 21489000 & 18869000 & 22431000 & $18.34 \%$ \\
\hline
\end{tabular}



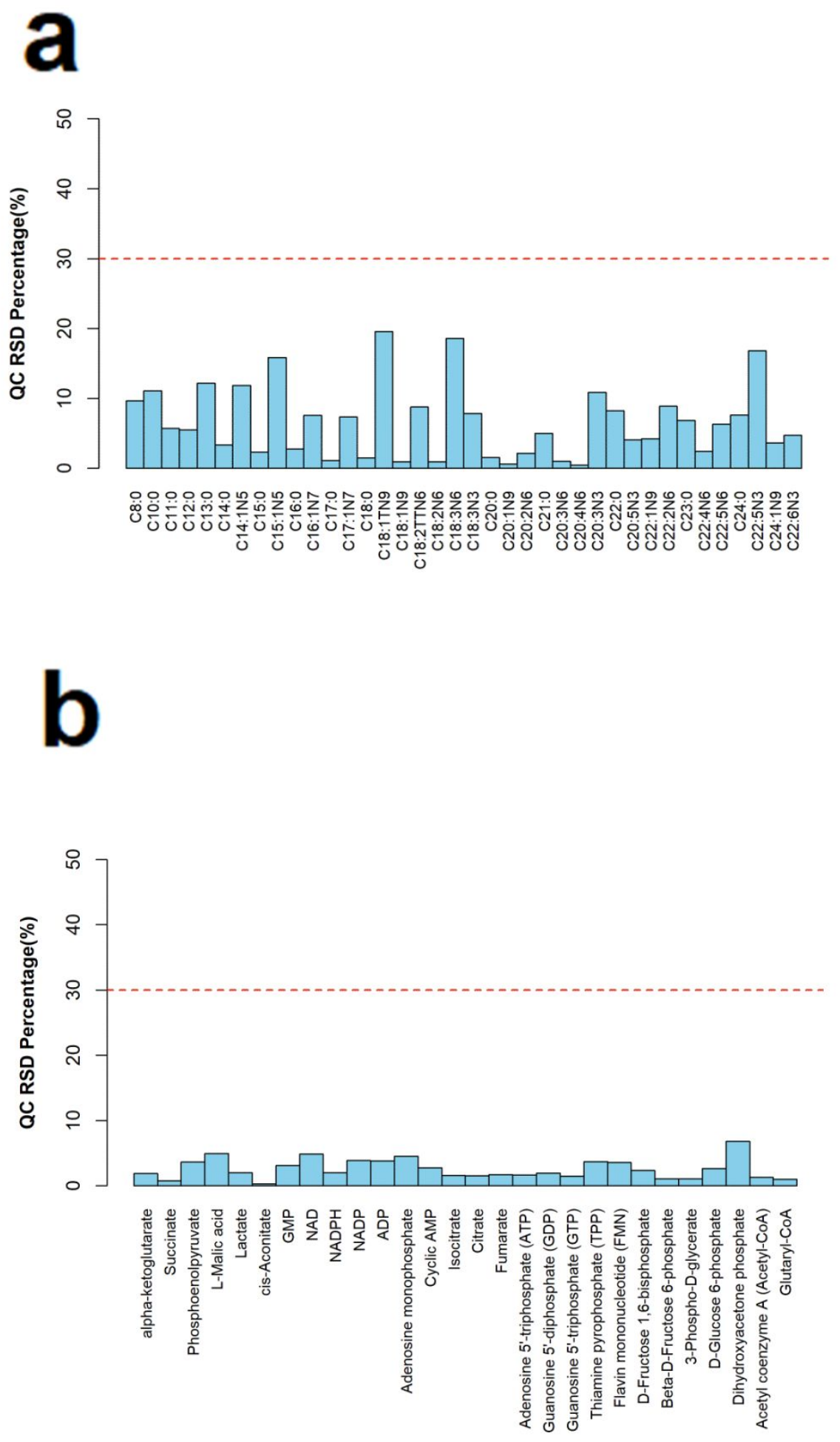

Supplementary Figure S1. (a, b). RSD (\%) of the quality control (QC) samples (fatty acids, nucleotides and energy metabolism). 
a

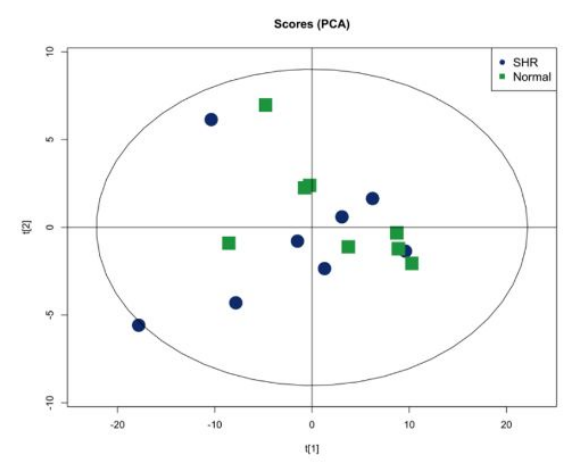

C

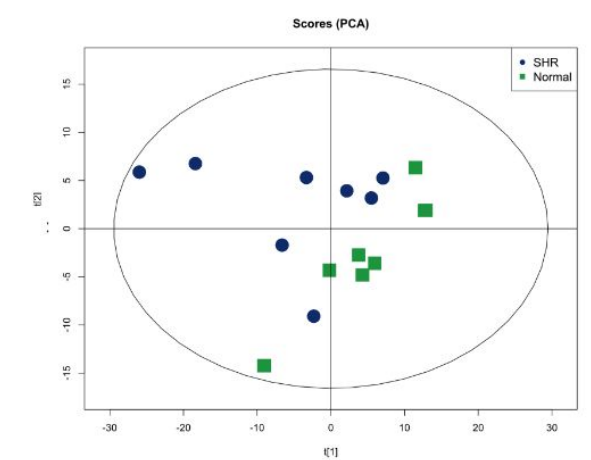

b

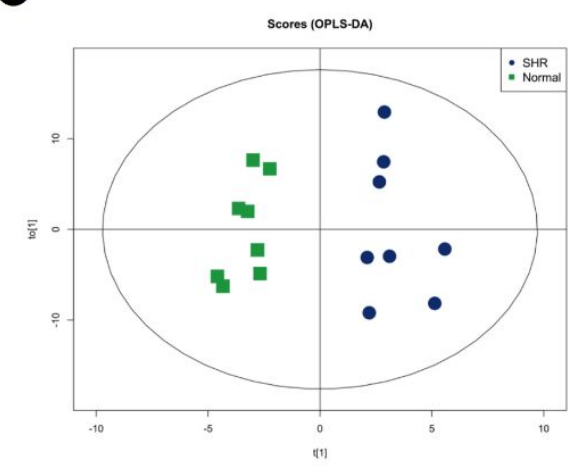

d

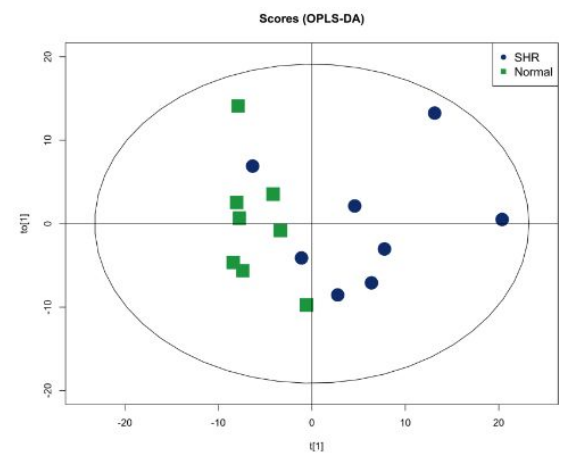

Supplementary Figure S2. Plots of PCA and OPLS-DA analysis between SHRs and normotensive rats. a. PCA based on 40 fatty acids; b. OPLS-DA based on 40 fatty acids; c. PCA based on 32 chemicals of nucleotides and energy metabolism; d. OPLS-DA based on 32 chemicals of nucleotides and energy metabolism. 
DECR1 IPI: IPI00213659

Unique peptide: FNIIQPGPIK

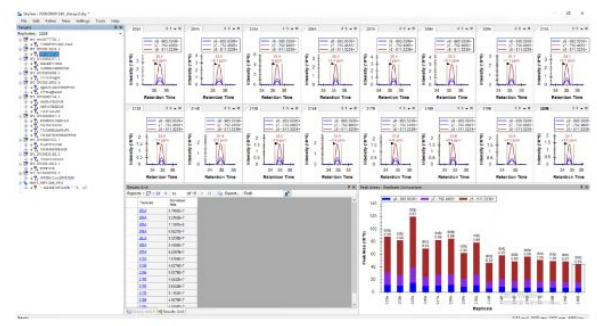

RHOA IPI: IPI00201699

Unique peptide: IVIVGDGACGKK

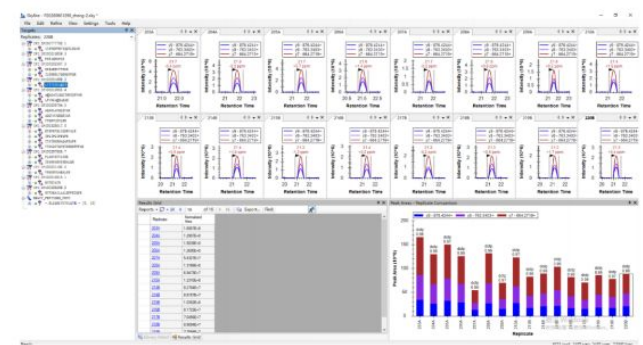

PADI2 IPI: IPI00202307

Unique peptide: ILSNESLTQENQYFQR

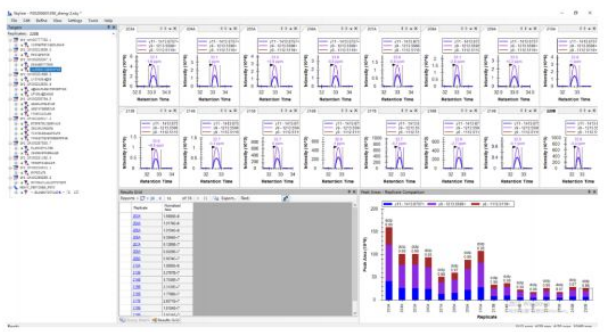

GNB1 IPI: IPI00212655

Unique peptide: LFVSGACDASAK

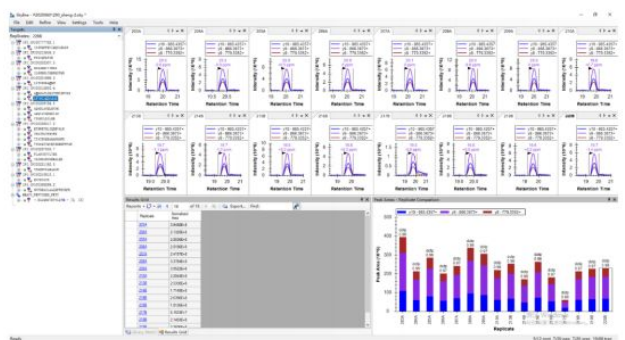

RHOB IPI:IPI00210815

Unique peptide: EVFETATR

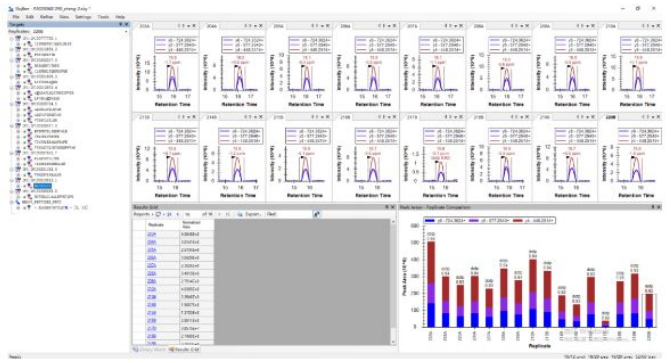

Supplementary Figure S3. The chromatograms of PRM analysis for the five proteins. 